\title{
DGI-Praxistage 2016
}

\section{Alexander Loth* \\ Die Notwendigkeit einer modernen Datenstrategie im Zuge der digitalen Transformation}

DOI 10.1515/iwp-2017-0014

Traditionell sind es die Mitarbeiter, die gute (oder zumindest glückliche) Entscheidungen treffen, die die Karriereleiter innerhalb von Unternehmen erklimmen. Und diese Kultur des Respekts gegenüber guten Instinkten durchdringt auch heutzutage noch die Entscheidungsfindung in vielen Unternehmen. In manchen Fällen werden Berater hinzugezogen, um unliebsamen oder rein präferentiellen Entscheidungen den Anschein externer Validierung zu geben; in anderen Fällen verlässt man sich auf die Weisheit von Vorgesetzten, Mentoren oder Gurus. Es zeichnet sich jedoch ab, dass bei Entscheidungen, die ein Unternehmen von sich aus nicht ohne weiteres unterstützen kann, immer mehr das Kollektiv herangezogen wird und man sich bei Entscheidungsfindung und Problemlösung der Kraft großer Zahlen bedient.

In unserer digital vernetzten Welt fallen jeden Tag riesige Mengen von Daten an. Das exponentielle Wachstum der Menge an generierten Daten führt unweigerlich zur Transformation ganzer Geschäftsmodelle. Nur Unternehmen, die große Datenmengen aus unterschiedlichen Quellen in umsetzbare Erkenntnisse verwandeln können, werden langfristig wettbewerbsfähig bleiben. Dazu bedarf es einer modernen Strategie, die den Fokus auf Daten legt und weit über deren reine Erhebung hinausgeht. Eine unternehmensweite Bereitstellung von Advanced Analytics kann hier einen Wettbewerbsvorteil bedeuten, insbesondere wenn sie den Schwerpunkt darauf legt, die Mitarbeiter mit den richtigen analytischen Werkzeugen auszustatten. Sind diese Werkzeuge einfach zu verwenden und gut in die tägliche Arbeit integriert, lässt sich die Akzeptanz - und somit Wirkung - maximieren.

Die voranschreitende digitale Transformation liefert Daten über nahezu jede Facette unseres Tuns. Jeder Besuch eine Webseite, jeder Klick, jede Suchanfrage und jeder Einkauf wird protokolliert und entweder mit unserer virtuellen Identität (wenn wir angemeldet sind) verknüpft, oder in einem System gespeichert, das unsere Sitzung per

*Kontaktperson: Alexander Loth, Tableau Germany GmbH, An der Welle 4, 60322 Frankfurt am Main, E-Mail: aloth@tableau.com
Cookie oder digitalem Fingerabdruck verarbeitet. Sind diese Daten erst einmal erhoben, werden sie für gewöhnlich in Silos der einzelnen Funktionen (vertikales Silo), Abteilungen (horizontales Silo) oder sogar in individuellen Projektsilos abgelegt. Um aus diesen Daten eine wertvolle und nützliche Ressource zu machen, müssen wir diese Silos aufbrechen. Dem stehen allerdings oft Fragen zur Inhaberschaft, Regularien und Governance im Weg. Das Sammeln von Daten allein generiert aber keinen Mehrwert. Der tatsächliche Business Impact hängt davon ab, wie „smart“ die gewonnen Erkenntnisse sind. Und das wiederum wird von der Vollständigkeit der Advanced Analytics-Lösung (s. Abb. 1) und der Komplexität der eingesetzten Modelle bestimmt. Präskriptive und semantische Analysen sind unter Umständen nur sehr schwer umzusetzen, insbesondere wenn es zunächst gilt, semistrukturierte Daten - wie etwa Social Media-Streams - zu klassifizieren. Man darf über die Umsetzung komplexer Modelle nicht vergessen, die leichte Beute einzusacken: lässt man die quantitativen Informationen einfließen (beispielsweise Umsatzdaten), kann man die diagnostischen Möglichkeiten skalieren.

Für die meisten Entscheidungsträger besteht die Herausforderung nicht in einem Mangel an Daten oder Datenquellen, sondern dass die zur Verfügung stehenden Datenquellen oftmals unterschiedliche Ergebnisse liefern oder schlichtweg nicht geeignet sind, die jeweils anstehende Entscheidung sinnvoll zu unterstützen. Leider wird die Rolle der IT oft unterschätzt. Die größte Herausforderung für Entscheidungsträger im Analytics-Zeitalter ist die mittlerweile etablierte Sichtweise, dass Unternehmen ihrer eigenen Datenbasis, bestehend aus Interaktionen mit potentiellen Kunden, Kunden, Lieferanten und Partnern, weniger vertrauen können als externen Erkenntnisquellen. Unternehmen transformieren die Art und Weise, wie sie ihre Kundenanalyse angehen in vielen verschiedenen Aspekten. Dieser Wandel ist jedoch weder branchenübergreifend, noch von Unternehmen zu Unternehmen innerhalb einer Branche konsistent. Ein Schlüssel zum Erfolg, bei dem die IT eine entscheidende Rolle spielen kann, ist den Faktor Kundenwissen möglichst gewinnbringend in die eigene Unternehmensstruktur zu integrieren. 


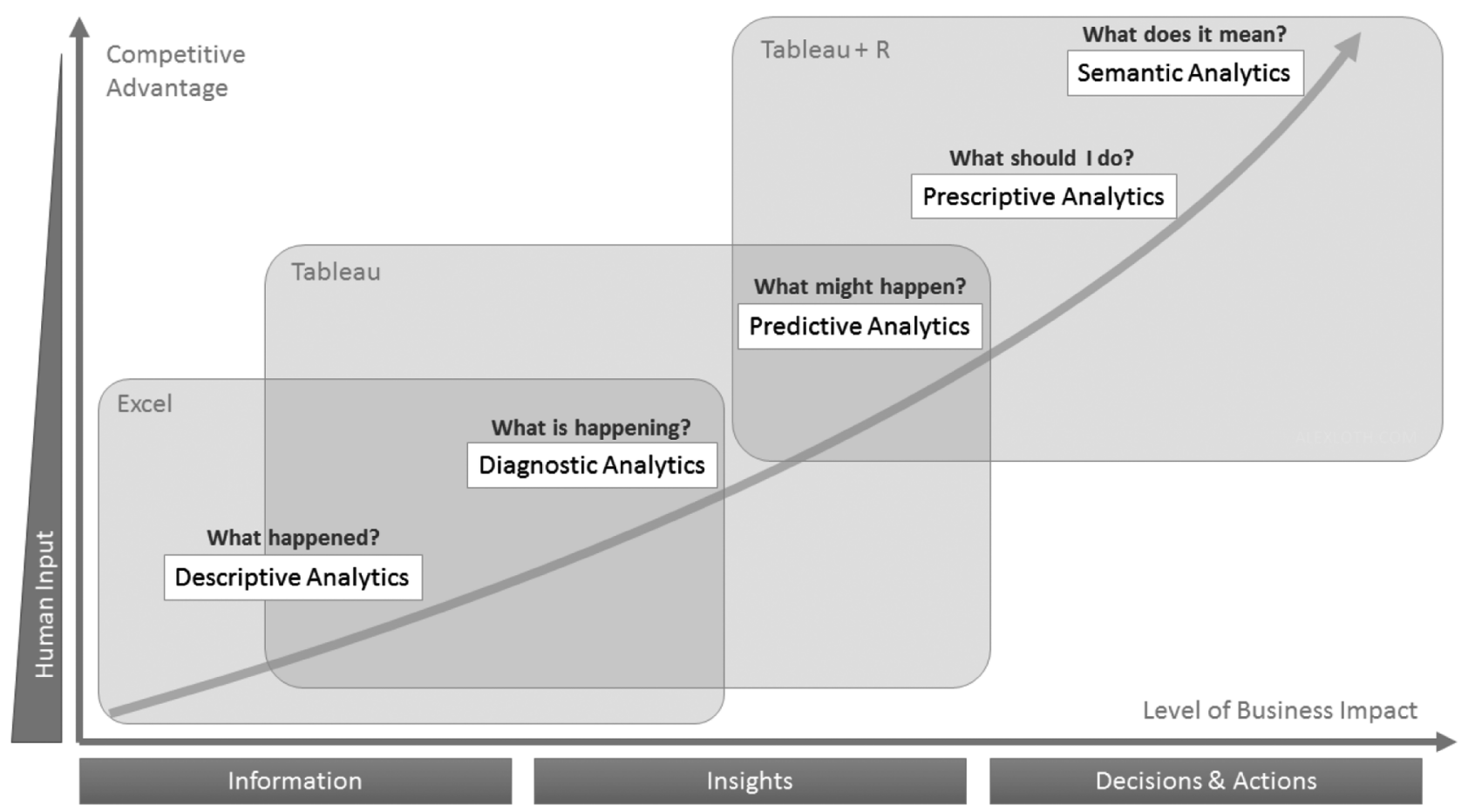

Abbildung 1: Loth-Modell des Reifegrads von Advanced Analytics.

Um eine datenzentrische Strategie zu entwickeln, muss man zunächst verstehen, wie ein Unternehmen Analysen durchführt und wie es mit den Ergebnissen umgeht: Welche Daten werden gesammelt, was passiert mit ihnen und wie wirken sie sich auf unsere Entscheidungen aus? Betrachtet man die Kunden aus einer taktischen oder strategischen Perspektive? Sieht man alle wichtigen Details? Wie kann man effizienter aggregieren? Sind die Analysen multidimensional oder sitzt man in Silos fest? Egal, wo sich ein Unternehmen im Spannungsfeld zwischen Berichtswesen und Data Discovery befindet, heutzutage müssen sich Analysetechniken nicht nur mit dem Business entwickeln, sie sollten dessen Evolution maßgeblich mitbestimmen. So gibt es beispielsweise Bereiche, in denen riesiges Potenzial zur Effizienzsteigerung durch Algorithmen und Maschinenlernen schlummert. Gleichzeitig werden manch andere Dinge immer das menschliche Auge erfordern. Wie viele Mitarbeiter im Unternehmen analysieren Daten? Und wie viele konsumieren die Ergebnisse? Es ist wichtig zu wissen, inwiefern die eingesetzte AnalysePlattform die analytische Arbeitslast und den Informationsfluss innerhalb des Unternehmens beeinflusst. Erst mit einem klaren Bild der Ausgangssituation lässt sich eine Strategie für den Wandel formulieren. In einer modernen Datenstrategie gibt es bei der Entscheidungsfindung keinen Schritt, der nicht von Analysen begleitet wird:

- Awareness: Erkennen, dass eine Entscheidung gefällt werden muss. Benachrichtigungen, Komplexe Ereig- nisverarbeitung und gut designte Dashboards bieten sich für diesen Zweck an.

- Scoping: Verstehen, welche Rahmenbedingungen wichtig und welche Bereiche indirekt betroffen sind. Statistik, Datenvisualisierung, Ad-hoc-Abfragen und bestimmte Data-Mining-Techniken eignen sich hierfür.

- Predicting: Identifizieren, wie sich unterschiedliche Handlungen auswirken. Simulation, Forecasting und Predictive Analytics sind in diesem Schritt der Entscheidungsfindung gefragt.

- Selecting: Die bestmögliche Handlung ausfindig machen. Hierbei können Optimierung und Regel-Engines helfen.

- Reporting: Überwachen der Auswirkungen einer Entscheidung. Hierbei ist selbstverständlich das Berichtswesen die relevante analytische Komponente.

Der erste Schritt auf dem Weg zu besserer Entscheidungsfindung im Unternehmen ist zu verstehen, wie gute (oder schlechte) Entscheidungen zustande kamen. Genau wie manche Unternehmen formale Prozesse für Aktivitäten haben, wie z.B. What-if-Analysen, prädiktive Wartung und Bestimmung von Abhängigkeiten in Korrelationen (s. Abb. 2), so müssen sie formale Prüfprozesse für Entscheidungen im gesamten Unternehmen einführen. Dies soll jedoch nicht dazu dienen, Beteiligte an negativen Entscheidungen für das Unternehmen zu bestrafen, sondern 


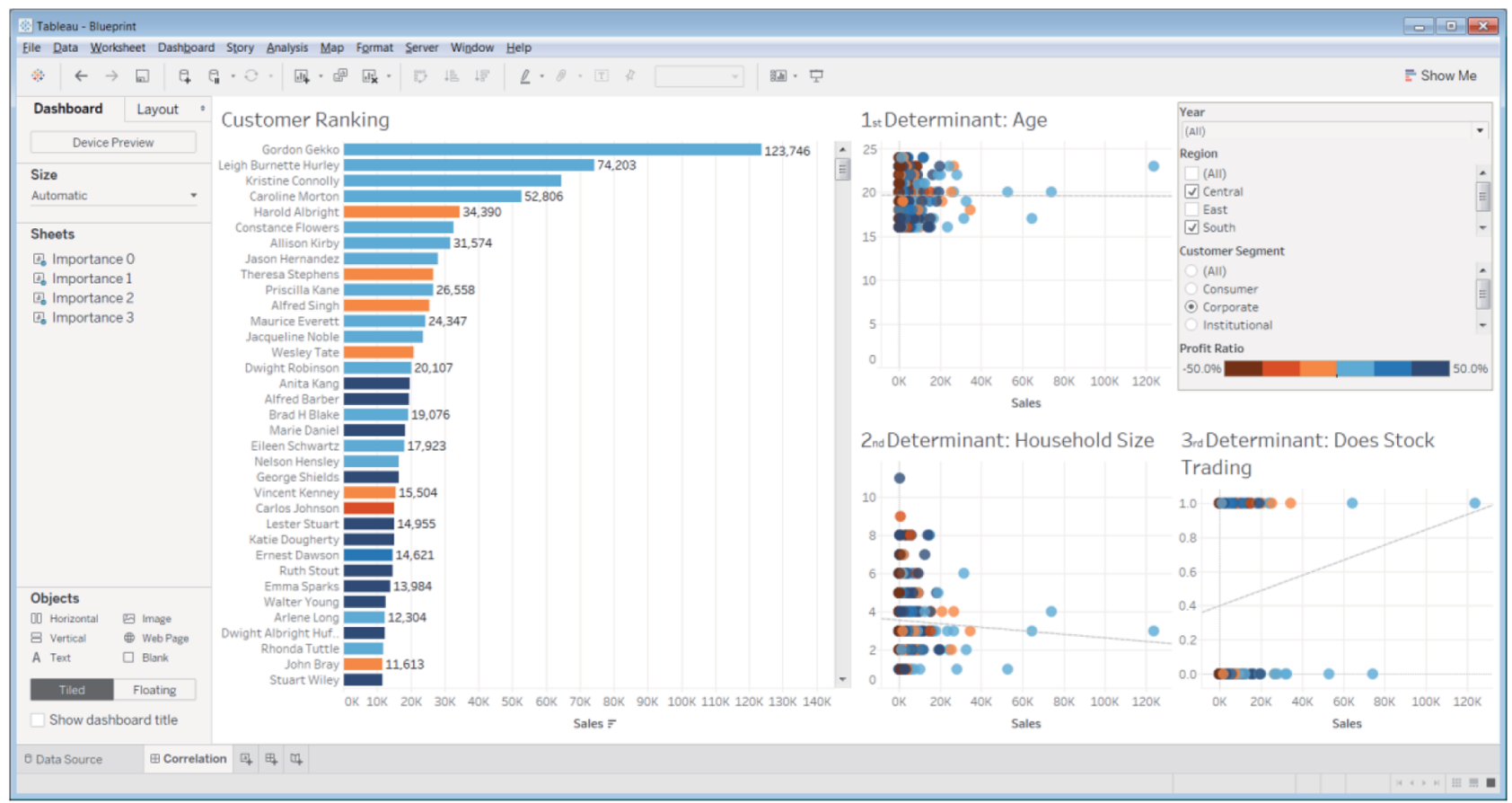

Abbildung 2: Interaktives Dashboard zur Darstellung von variablen Abhängigkeiten in Tableau.

den Entscheidungsfindungsprozess und -stil im Allgemeinen zu verbessern.

Die Rolle der IT nähert sich hierbei wieder ihren Wurzeln an. Statt eine Berichtsfabrik für den Rest des Unternehmens zu unterhalten, wird sie wieder zum Dienstleister und Partner, der Infrastruktur bereitstellt. IT-Mitarbeiter werden entlastet und erhalten den Freiraum, ihre professionelle Energie und Kreativität in den Dienst der Innovation zu stellen, und die Mitarbeiter in den Abteilungen sehen ihre Datenfragen nicht am Flaschenhals Berichtswesen verhungern. Nur so lassen sich die Investitionen in Business Intelligence und Analytics optimal in den Dienst der strategischen Ziele des Unternehmens stellen.

Moderne Unternehmen sehen sich vielen analytischen Anforderungen gegenüber, und diese Anforderungen werden unweigerlich schneller wachsen, als Unternehmen sie bedienen können. Es ist daher unerlässlich, Analytics als lebenswichtigen Teil der eigenen Datenstrategie zu verstehen und entsprechend zu planen.

Dabei ist ein umfassender Betrachtungswinkel sinnvoll, denn die wachsende Nachfrage nach Analysen und Erkenntnissen wird mehr und mehr von den kundenbezogenen Abteilungen wie Marketing oder Support ausgehen. Dementsprechend wird auch das Budget für Analytics verstärkt aus diesen Abteilungen kommen, statt aus einem zentralisierten IT- oder BI-Budget. Dort, wo viele Kundendaten vorhanden sind, wird der CMO bald mehr für Analytics ausgeben als der CIO. Und dort, wo Mitarbeiter über gut integrierte, intuitive Werkzeuge für komplexe Ana- lysen verfügen, können gute Instinkte und datenbasierte Entscheidungen Hand in Hand für den Erfolg sorgen.

Deskriptoren: Datenanalyse, Entscheidungshilfe, Prognose, Innerbetriebliche Information

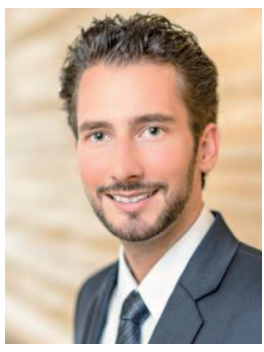

Alexander Loth

Tableau Germany GmbH

An der Welle 4

60322 Frankfurt am Main

aloth@tableau.com

Alexander Loth ist seit 2015 Sales Consultant bei Tableau und hat mehr als neun Jahre Erfahrung im Bereich Enterprise Software mit Fokus auf Data Strategy, Data Science, und Business Analytics. Er hilft seinen Klienten beim Aufbau einer datenzentrischen Strategie. Bevor er Teil der Tableau-Familie wurde, war Alexander Loth als Data Scientist am CERN, als Consultant bei Capgemini und im Software Engineering bei SAP tätig. Zudem hat er einen MBA von der Frankfurt School of Finance \& Management. 